\title{
Electric Relaxation in $\mathrm{Nb}_{6} \mathrm{VSb}_{3} \mathrm{O}_{25}$-Ceramics
}

\author{
T. Groń ${ }^{a}$, E. FilipeK ${ }^{b}$, M. Piz $^{b}$, Z. Kukula ${ }^{a}$ And S. PAWlus ${ }^{a, c}$ \\ ${ }^{a}$ Institute of Physics, University of Silesia, Uniwersytecka 4, 40-007 Katowice, Poland \\ ${ }^{b}$ Department of Inorganic and Analytical Chemistry, West Pomeranian University of Technology, \\ al. Piastów 42, 71-065 Szczecin, Poland \\ ${ }^{c}$ Silesian Center for Education and Interdisciplinary Research, University of Silesia, \\ 75 Pułku Piechoty 1A, 41-500 Chorzów, Poland
}

(Received November 22, 2015; in final form February 19, 2016)

\begin{abstract}
Broadband dielectric spectroscopy measurements of $\mathrm{Nb}_{6} \mathrm{VSb}_{3} \mathrm{O}_{25}$ showed that in both the real $(\varepsilon /)$ and imaginary $(\varepsilon \prime \prime)$ components of permittivity there is visible relaxation process strongly obscured by dc conductivity. Application of the electric modulus representation of the data enables to study temperature evolution of this relaxation together with conductivity relaxation. It was showed that the activation energies for both processes are close. Low-frequency loss tangent increases strongly with temperature, suggesting that in the compound under study additional energy losses are associated with the conduction of electric current, as determined by the JouleLenz law.
\end{abstract}

DOI: 10.12693/APhysPolA.129.355

PACS/topics: 77.22.Ch, 77.22.Gm, 77.84.Bw

\section{Introduction}

Recent studies concerning the reactivity in the solid state among $\mathrm{V}_{2} \mathrm{O}_{5}, \mathrm{Nb}_{2} \mathrm{O}_{5}$ and $\alpha-\mathrm{Sb}_{2} \mathrm{O}_{4}$ showed that those oxides interact in air, producing apart from $\mathrm{Nb}_{2} \mathrm{VSbO}_{10}$ [1-3], also new compound with formula $\mathrm{Nb}_{6} \mathrm{VSb}_{3} \mathrm{O}_{25}$ [4]. It can find application as the component of gas sensors and element of catalysts in reactions of removing hydrogen by oxidizing of light hydrocarbons. In $\mathrm{Nb}_{2} \mathrm{VSbO}_{10}$ [5], as well as in $\mathrm{Nb}_{6} \mathrm{VSb}_{3} \mathrm{O}_{25}$, antimony, niobium, and vanadium are mainly in +5 oxidation state. Preliminary studies of $\mathrm{Nb}_{6} \mathrm{VSb}_{3} \mathrm{O}_{25}$ by EPR, showed that the total content of $\mathrm{V}^{4+}+\mathrm{Nb}^{4+}$ does not exceed $1.3 \%$. In the solid state new compound is stable in air up to $\approx 1200 \mathrm{~K}$ and next melts with deposition of solid $\mathrm{Nb}_{9} \mathrm{VO}_{25}$.

The electrical measurements performed on $\mathrm{Nb}_{6} \mathrm{VSb}_{3} \mathrm{O}_{25}$-ceramics [6], showed a sharp increase of the $n$-type electrical conduction at $330 \mathrm{~K}$ from the extrinsic region with the activation energy of $E_{\mathrm{a}}=0.01 \mathrm{eV}$ to the intrinsic one with the activation energy of $E_{\mathrm{a}}=0.75 \mathrm{eV}$. At high temperatures, i.e. above $520 \mathrm{~K}$, a rather well defined linear slope of diffusion thermopower was found which extrapolates to $(0,0)$. The $I-V$ characteristics, measured at 300 and $400 \mathrm{~K}$, showed a symmetric and non-linear behaviour as well as a broad minimum of the conductance $G$ vs. the applied voltage $V$, which is being shifted into the higher voltages and simultaneously its value falls with a rise in temperature [6].

In this contribution we present the broadband dielectric spectroscopy of $\mathrm{Nb}_{6} \mathrm{VSb}_{3} \mathrm{O}_{25}$-ceramics studied in a wide range of temperature and frequency. The electric modulus formalism is applied to analyse the dielectric data.

\section{Experimental}

Polycrystalline compound $\mathrm{Nb}_{6} \mathrm{VSb}_{3} \mathrm{O}_{25}$ was synthesized by solid state reaction method as described earlier $[4,6] . \mathrm{Nb}_{6} \mathrm{VSb}_{3} \mathrm{O}_{25}$ has been obtained by heating of the mixture $\mathrm{V}_{2} \mathrm{O}_{5} / \mathrm{Nb}_{2} \mathrm{O}_{5} / \mathrm{Sb}_{2} \mathrm{O}_{4}$ in molar ratio 1:6:3 in $24 \mathrm{~h}$ stages in air in the temperature range from 870 to $1120 \mathrm{~K}$ [6]. $\mathrm{Nb}_{6} \mathrm{VSb}_{3} \mathrm{O}_{25}$ has been characterized by the methods: XRD (diffractometer EMPYREAN II, PANalytical, using the radiation $\mathrm{Cu} K_{\alpha}$, filter $\mathrm{Ni}$ ) and Novocontrol Alpha Impedance analyzer using pellets, polished and sputtered with $(\approx 80 \mathrm{~nm}) \mathrm{Ag}$ electrodes in the frequency and temperature range $10^{-2}-10^{6} \mathrm{~Hz}$ and 233-373 K, respectively, for real $(\varepsilon \prime)$ and imaginary $(\varepsilon \prime \prime)$ components of permittivity as well as loss tangent $(\tan \delta)$ measurements. For the dielectric measurements, the powder samples were compacted in a disc form $(10 \mathrm{~mm}$ in diameter and 1-2 mm thick) using a pressure of $1.5 \mathrm{GPa}$ and then they were sintered during $2 \mathrm{~h}$ at $873 \mathrm{~K}$.

\section{Results and discussion}

The powder diffraction pattern of obtained single phase sample containing of $\mathrm{Nb}_{6} \mathrm{VSb}_{3} \mathrm{O}_{25}$ was indexed in order to confirm the system in which the compound crystallizes and to determine parameters of the unit cell. The results of this part of the study were almost identical to those described in [6], and revealed that $\mathrm{Nb}_{6} \mathrm{VSb}_{3} \mathrm{O}_{25}$ crystallized in orthorhombic system. The orthorhombic unit cell parameters are: $a=0.34912 \mathrm{~nm}, b=$ $0.67119 \mathrm{~nm}, c=1.79974 \mathrm{~nm}$. The volume of a unit cell is $V=0.416128 \mathrm{~nm}^{3}$, and the number of stoichiometric units per one unit cell is $Z=1[6]$. On the basis of differential thermal analysis-thermogravimetric (DTATG) results $\mathrm{Nb}_{6} \mathrm{VSb}_{3} \mathrm{O}_{25}$ was confirmed to melts in air at $\approx 1200 \mathrm{~K}[4,6]$. A scanning electron microscopy (SEM) image of a polycrystalline $\mathrm{Nb}_{6} \mathrm{VSb}_{3} \mathrm{O}_{25}$ is also very similar to the image of the compound obtained previously. The crystals are of very irregular plate-like shape and have sizes from $\approx 0.5 \mathrm{~mm}$ to several $\mathrm{mm}[6]$.

It was well known fact that relaxation phenomena in different materials were strongly dependent on the 


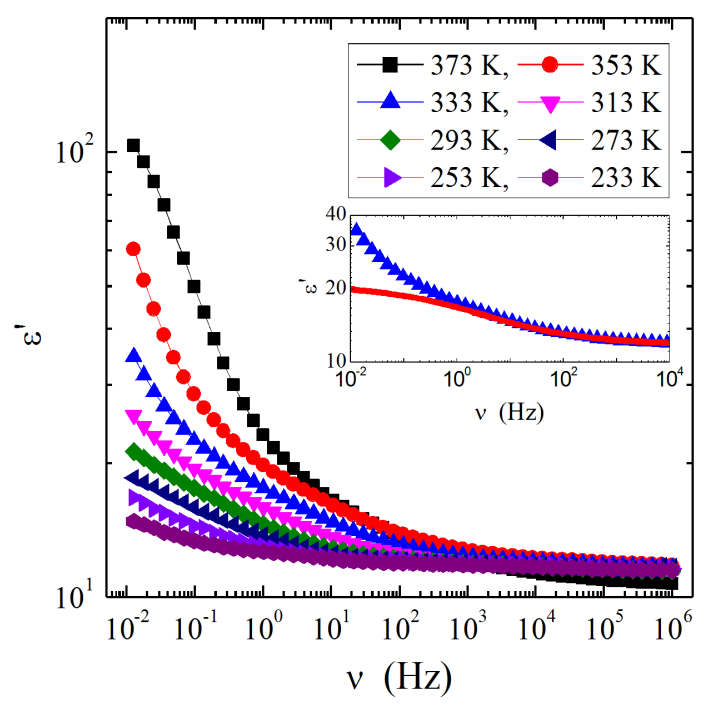

Fig. 1. Real component of permittivity $\varepsilon$ ' vs. frequency $\nu$ in the temperature range $233-373 \mathrm{~K}$. The inset presents selected spectrum frequency region at $333 \mathrm{~K}$ in which relaxation process is observed (depicted by red solid line).

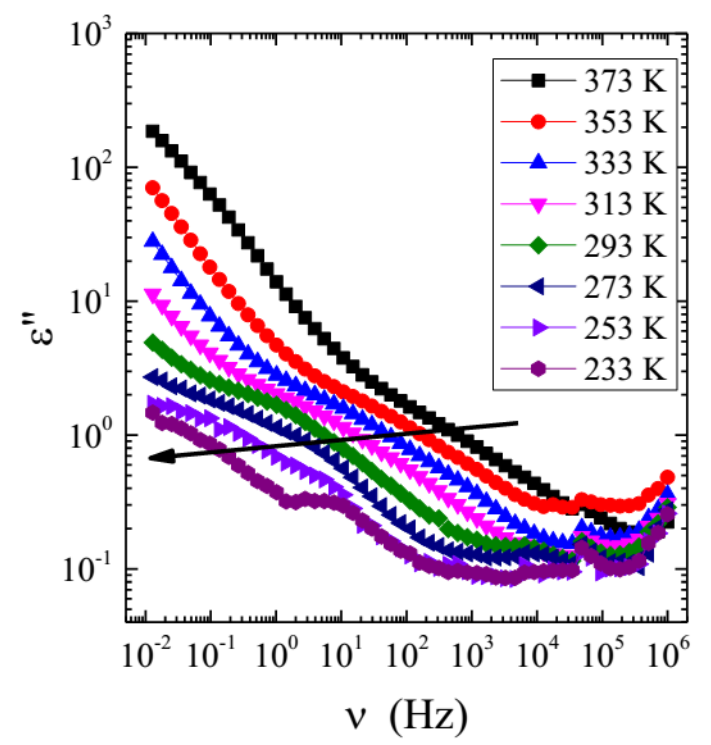

Fig. 2. Imaginary component of permittivity $\varepsilon / \prime$ vs. frequency $\nu$ in the temperature range $233-373 \mathrm{~K}$. The arrow indicates direction of temperature evolution of the relaxation process.

temperature conditions. These changes take place in extremely wide time range from ns up to millions of seconds [7]. Excellent experimental tool for study of their behavior in this time span is broadband dielectric spectroscopy. In the frequency domain, the dielectric response of the system subjected to an external oscillating electric field is fully characterized by the complex permittivity, $\varepsilon^{*}(2 \pi \nu)=\varepsilon \prime(2 \pi \nu)+\mathrm{i} \varepsilon \prime \prime(2 \pi \nu)$, where the real and imaginary components are the storage and loss, respectively, of the energy during each cycle of the electric field [8]. Frequency spectra of both, real and imaginary parts of complex dielectric permittivity, exhibit existence of relaxation process (see Fig. 1, red solid line in the inset of Fig. 1 and Fig. 2). For many materials like glass forming liquids or polymers this relaxation is usually clearly visible as step function or peak for real and imaginary parts of permittivity, respectively. However, in the case of presented sample, like for many other ceramics and ionic liquids, analysis of the relaxation processes in quoted representation is difficult. The difficulties are related to significant dc-conductivity contribution that, at least partially obscure relaxation processes [9]. Only closer inspection of dielectric spectra in Figs. 1 and 2 reveals a step-like frequency dependence of the real part of the dielectric permittivity, $\varepsilon \prime(2 \pi \nu)$ and the inset of Fig. 1, as well as the departure of $\varepsilon \prime \prime(2 \pi \nu)$ from the $\nu^{-1}$ dependence. The upward trend, visible in the loss spectra at low frequency region (below $c a .100 \mathrm{~Hz}$ ) is attributed entirely to the dc-conductivity. This suggests that in the compound under study additional energy losses are associated with the conduction of electric current, as determined by the Joule-Lenz law.

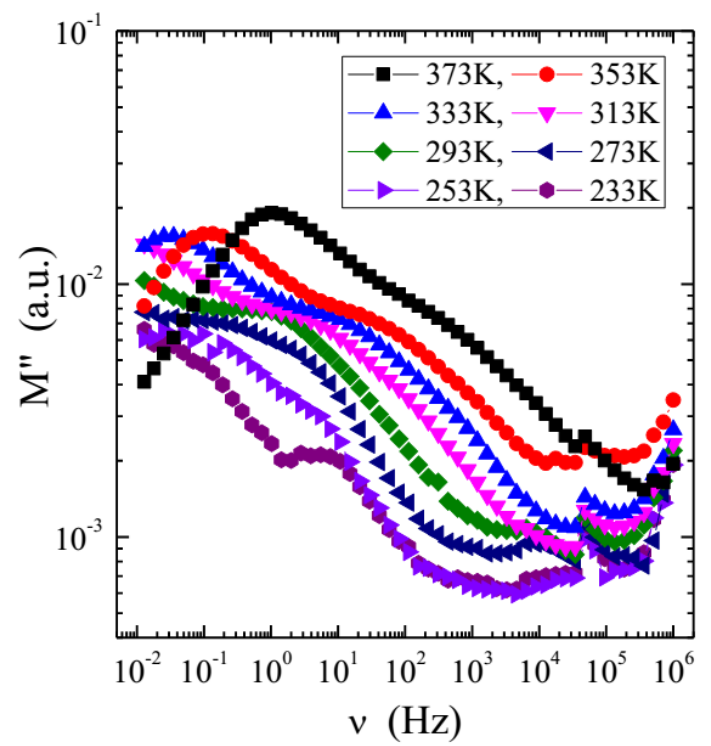

Fig. 3. Electrical modulus $M \prime \prime$ vs. frequency $\nu$ in the temperature range $233-373 \mathrm{~K}$.

In the case of $\varepsilon \prime$, similar effect from electrode polarization masks partially relaxation process in low frequency area. However, for both representations $\varepsilon l$ and $\varepsilon / l$ it is clear that time scale of relaxation process becomes longer with decreasing temperature. The effect of conductivity can be suppressed when data are presented in the electrical modulus representation that is reciprocal of complex dielectric permittivity [8]:

$$
M^{*}=1 / \varepsilon^{*}=M \prime(2 \pi \nu)+\mathrm{i} M \prime \prime(2 \pi \nu) .
$$

The dielectric loss curves in Figs. 3 and 4 were portrayed by a sum of the Cole-Cole distribution function, namely [10]: 


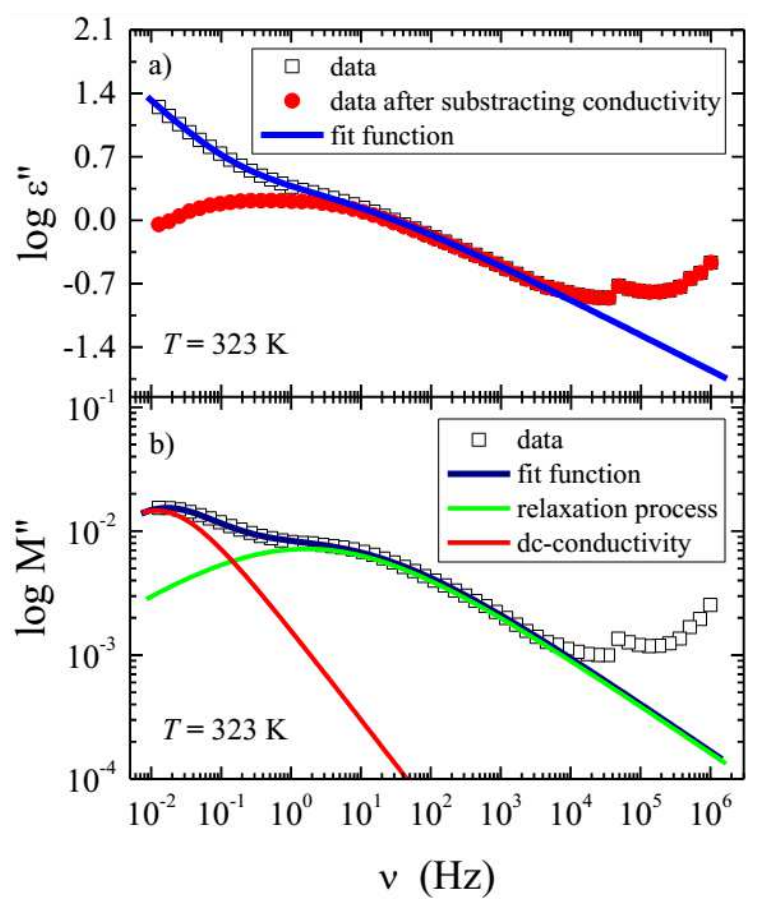

Fig. 4. (a) Selected spectrum measured at $323 \mathrm{~K}$ (empty squares) and without conductivity part (filled red circles). (b) Modulus spectrum for the same temperature. Two solid curves represent dc-conductivity (red) and relaxation process (green), respectively.

$$
\varepsilon *=\varepsilon_{\infty}+\frac{\Delta \varepsilon}{1+(\mathrm{i} 2 \pi \nu \tau)^{1-a}},
$$

where $\Delta \varepsilon=\varepsilon_{0}-\varepsilon_{\infty}$ is the dielectric strength, $\varepsilon_{\infty}$ represents the asymptotic value of permittivity at high frequencies, $\varepsilon_{0}$ is the value of the opposite limit, and $\tau$ is the relaxation time equal to the maximum relaxation time $\tau_{\max }$. Usually, the relaxation time is determined as the inverse of the maximum peak frequency, $\tau=1 /\left(2 \pi \nu_{\max }\right)$ [8]. The power exponent $c=1-a$ is responsible for symmetric broadening of the loss curve and term describing contribution from dc-conductivity: $\sigma_{\mathrm{dc}} / \varepsilon_{0} 2 \pi v$. The electric modulus data were described by a sum of two Cole-Cole functions, one describing peak from the dc-conductivity and second - for fitting the relaxation process.

From the physical viewpoint, the electrical modulus corresponds to the relaxation of the electric field in the material when the electric displacement remains constant. Therefore, the electrical modulus depicts the real dielectric process [11-13]. As can be seen in Figs. 3 and 4, data presented in this way exhibit two pronounced relaxation peaks. Slower of them is Debye-like in shape and related to the dc-conductivity. Faster, wider and with lower amplitude is related to relaxation process observed in dielectric permittivity spectra. Both processes move toward lower frequencies with cooling.

Based on the data presented in Fig. 5, the activation energy $E_{\mathrm{a}}$ was determined for both processes using the formula $[14,15]$ :

$$
\tau=\tau_{0} \exp \left(\frac{E_{\mathrm{a}}}{k T}\right),
$$

where $k$ is the Boltzmann constant and $\tau_{0}$ is the characteristic relaxation time which is defined as the reciprocal of the frequency of the hopping of potential barrier $[14,15]$. The values of the activation energy estimated in the temperature range $320-345 \mathrm{~K}$ from Eq. (3) are as follows: $0.60,0.63$, and $0.57 \mathrm{eV}$ for conductivity (modulus), relaxation (permittivity) and relaxation (modulus), respectively. These values are close to the activation energy $E_{\mathrm{a}}=0.75 \mathrm{eV}$, which has been estimated from the temperature dependence of the electrical conductivity in the intrinsic region, i.e. in the temperature range $375-398 \mathrm{~K}[6]$.

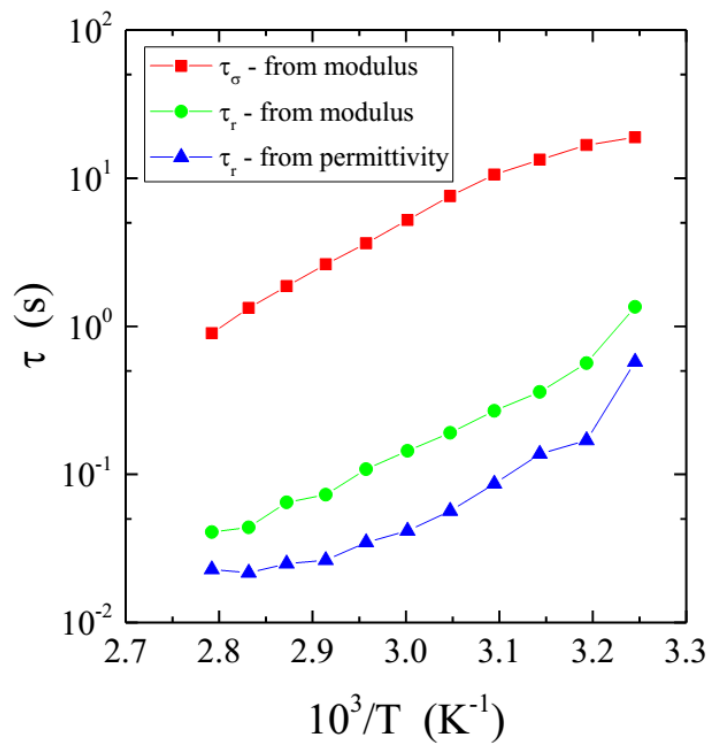

Fig. 5. Arrhenius plot of relaxation times $\tau$ vs. reciprocal temperature $\left(10^{3} / T\right)$ for both dc-conductivity and relaxation processes.

The temperature behaviour of $\tau$ for all relaxation processes and for dielectric permittivity and electric modulus representations are depicted in Fig. 5. It is obvious that time scale of the conductivity relaxation time, $\tau_{\sigma}$ is $c a$. two order of magnitude slower than for the relaxation process $\tau_{\mathrm{r}}$, however, similar slopes of both dependences indicate on close activation energies for both processes. The values of the dielectric and modulus relaxation times, $\tau_{\mathrm{r}}$, are related with some shifting factor dependent e.g. on static and infinity permittivity values [13]. Dielectric measurements of the real and imaginary components of permittivity as well as the loss tangent showed that the majority charge carriers must be partially recombined in the shallow trapping centres [16], lying under the bottom of the conduction band. A natural source of these traps can be the grain-boundaries with the depletion layers of the adjacent grains. Similar behaviour was observed for $\mathrm{ZnO}$ varistors [17] and $\mathrm{Nb}_{2} \mathrm{VSbO}_{10}$-ceramics [18] as well as some novel copper/cobalt and rare-earth metal tungstates [19]. 
In conclusion, the dc-conductivity and relaxation processes dominate in $\mathrm{Nb}_{6} \mathrm{VSb}_{3} \mathrm{O}_{25}$ with comparable activation energies for both ones. The former is $c a$. twice slower than the other.

\section{Acknowledgments}

The authors are grateful to the team workshop of the Institute of Physics for providing practical and technical assistance. This work was partly supported by Ministry of Scientific Research and Information Technology (Poland) and funded from science resources: No. 1S0300-500-1-05-06.

\section{References}

[1] E. Filipek, M. Piz, J. Therm. Anal. Calorim. 101, 447 (2010).

[2] E. Filipek, M. Piz, Patent RP No. 213420 B1 (2013).

[3] M. Ziółek, H. Golińska-Mazwa, E. Filipek, M. Piz, Catal. Today 187, 159 (2012).

[4] E. Filipek, M. Piz, J. Alloy. Compd. 661, 141 (2016).

[5] J. Typek, G. Zolnierkiewicz, A. Cyran, K. Wardal, E. Filipek, M. Piz, in: Proc. Conf. FNMA'11, IMIM'11, AUXETICS'11, Eds. J. Rybicki, K.W. Wojciechowski, Task Publishing, Gdańsk 2011, Abstract book, P62, 2011, p. 181.
[6] T. Groń, E. Filipek, M. Piz, H. Duda, Mater. Res. Bull. 51, 105 (2014).

[7] U. Schneider, P. Lunkenheimer, R. Brand, A. Loidl, Phys. Rev. E 59, 6924 (1999).

[8] A. Molak, M. Paluch, S. Pawlus, J. Klimontko, Z. Ujma, I. Gruszka, J. Phys. D Appl. Phys. 38, 1450 (2005).

[9] P.B. Macedo, C.T. Moynihan, R. Bose, Phys. Chem. Glasses 13, 171 (1972).

[10] K.S. Cole, R.H. Cole, J. Chem. Phys. 9, 341 (1941).

[11] H. Wagner, R. Richert, Polymer 38, 5801 (1997).

[12] C. Leon, M.L. Lucia, J. Santamaria, Phys. Rev. B 55, 882 (1997).

[13] R. Richert, H. Wagner, Solid State Ion 105, 167 (1998).

[14] N.F. Mott, E.A. Davis, Electronic Processes in NonCrystalline Materials, Clarendon Press, Oxford 1971.

[15] Encyclopaedia of Modern Physics, Ed. A.K. Wróblewski, PWN, Warsaw 1983 (in Polish).

[16] J.G. Simmons, Phys. Rev. 155, 657 (1967).

[17] C. Li, J. Wang, W. Su, H. Chen, W. Wang, D. Zhuang, Physica B 307, 1 (2001).

[18] T. Groń, E. Filipek, M. Piz, H. Duda, T. Mydlarz, Mater. Res. Bull. 48, 2712 (2013).

[19] T. Groń, E. Tomaszewicz, Z. Kukuła, S. Pawlus, B. Sawicki, Mater. Sci. Eng. B 184, 14 (2014). 